Tropical Journal of Pharmaceutical Research May 2021; 20 (5): 899-910

ISSN: $1596-5996$ (print); 1596-9827 (electronic)

(C) Pharmacotherapy Group, Faculty of Pharmacy, University of Benin, Benin City, 300001 Nigeria.

\title{
LncRNA-NEAT1/miR-148a-3p axis regulates cell viability, apoptosis and autophagy through wnt/ $\beta$-catenin signaling pathway in Breast Cancer
}

\author{
Song An, Yuren Xia, Zilu Gao, Xiaoxuan Sun, Jian Wang* \\ Department of Comprehensive Surgery, Tianjin Medical University Cancer Institute and Hospital, National Clinical Research \\ Center for Cancer, Key Laboratory of Breast Cancer Prevention and Therapy, Tianjin Medical University, Ministry of Education, \\ Tianjin, China
}

*For correspondence: Email: vby4585@126.com

Sent for review: 02 May 2020

Revised accepted: 20 June 2020

\begin{abstract}
Purpose: Breast cancer has over the years been one of major acute carcinomas in women. This study investigated the fundamental mechanistic functions of the IncRNA-NEAT1/miR-148a-3p/Wnt/ß-catenin axis in moderating cell viability, apoptosis and autophagy in Breast Cancer (BC).

Methods: RT-qPCR measured expression of IncRNA NEAT1 and microRNA-148a-3p in human cell lines for Breast Cancer. Cell transfection upregulated or silenced the genes with CCK-8, western blot and FCM apoptosis assays determining the cellular growth, proliferation and protein expression related to autophagy biomarkers. Furthermore, luciferase assay analyzed the luciferase activity of IncRNANEAT1 and microRNA-148a-3p

Results: The outcomes indicated that LncRNA-NEAT1 was upregulated in BC cell lines and promoted cell viability, autophagy and inhibited Apoptosis in BC cells. However, IncRNA-NEAT1 knockdown inhibited cell viability, autophagy and enhanced apoptosis. In addition, IncRNA-NEAT1 directly targeted microRNA-148a-3p. And, it was found that microRNA-148a-3p overturns the cellular viability, autophagy and inhibitory effects on Apoptosis imposed by IncRNA-NEAT1 overexpression. Lastly, overexpressed IncRNA-NEAT1 activated the Wnt/ $\beta$-catenin regulatory network through sponging microRNA-148a-3p in $B C$ cell lines.

Conclusion The present study showcased that IncRNA-NEAT1 could enhance tumor development in breast cancer via playing the role of molecular sponge to microRNA-148a-3p, and eventually hyper invigorating the Wnt/ $\beta$-catenin regulatory network.
\end{abstract}

Keywords: IncRNA-NEAT1; miR-148a-3p; Cellular Viability; Apoptosis; Autophagy; Breast Cancer

\begin{abstract}
This is an Open Access article that uses a fund-ing model which does not charge readers or their institutions for access and distributed under the terms of the Creative Commons Attribution License (http://creativecommons.org/licenses/by/4.0) and the Budapest Open Access Initiative (http://www.budapestopenaccessinitiative.org/read), which permit unrestricted use, distribution, and reproduction in any medium, provided the original work is properly credited.

Tropical Journal of Pharmaceutical Research is indexed by Science Citation Index (SciSearch), Scopus, International Pharmaceutical Abstract, Chemical Abstracts, Embase, Index Copernicus, EBSCO, African Index Medicus, JournalSeek, Journal Citation Reports/Science Edition, Directory of Open Access Journals (DOAJ), African Journal Online, Bioline International, Open-J-Gate and Pharmacy Abstracts
\end{abstract}

\section{INTRODUCTION}

Breast Cancer (BC) is one of the malignant illness prevalent among women across the globe and is very fatal with an approximated 2.4 million fresh incidences and 523000 fatalities registered in 2015 [1]. In the USA, the American Cancer Society gave a summary of breast cancer statistics among women and it was approximated that by 2013 about 39,620 breast cancer deaths 
were foreseen to happen, commonly among women of 40 years and older [2]. In China, a 5year occurrence information shows that roughly $11 \%$ of global BC takes place in China and that the prevalence has quickly become amplified than before over the past decades between 20$30 \%$ [3]. For instance, the China National Cancer Centre (2011) registered 248,620 fresh BC incidences among women, consistent with agestandardized occurrence rate at 29 for every 100,000 Chinese females. Recently, Ye et al., established epidemiologic factors in BC and corresponding subtypes among Chine females. In their study, they concluded that postmenopausal females had low risk of having BC but possessed non-luminal subtype, while females having an account of abortion had very high risk of having $\mathrm{BC}$ [4]. However, females having large breast density and Breast Imaging Reporting and Data System (BIRADS) scores of injuries were disposed to having advanced stage BC [4]. Furthermore, diagnosis, prognosis, prediction of therapy effectiveness and toxicity and reoccurrence have been hot subjects in recent years [5]. Thus, deaths related to $B C$ have declined due to application of adjuvant chemotherapeutic and hormonal agents, employing biomarkers as a technique to help in diagnosing breast cancer, conducting prognosis, forecasting of therapeutic reaction, and disease examination throughout and post-therapy [5]. In addition, these diagnostic platforms and imaging approaches provide the base for conducting diagnostic examination viz; mammography, magnetic resonance imaging (MRI), positronemission tomography (PET), Computed tomography (CT), and single-photon emission computed tomography (SPECT) that offer useful data related to $\mathrm{BC}$ cases [6]. Likewise, use of biochemical biomarkers for instance proteins, DNAs, mRNAs, and microRNAs has been widely accepted as novel diagnostic and treatment means such as radiation and chemo-therapy in BC patients [6].

Not long ago, scientists discovered the importance of utilizing long-non coding RNAs (IncRNAs) and miRNAs in diagnosis and treatment of breast cancer. Long noncoding RNAs (IncRNAs) are a class of non-encrypted RNAs comprising in excess of 200 nucleotides that act an essential role in numerous human cancers such as breast cancer [7], hepatoma [8], clear cell Renal cell carcinoma [9] and other cancers. In this study, we investigate the role of IncRNA-NEAT1/ miR-148a-3p in advancing breast cancer via several cellular functions such as viability, apoptosis and autophagy which still remain unclear. A large body of evidence shows that IncRNA-NEAT1 has been involved is various studies related to breast cancer but the functions of IncRNA-NEAT1/ miR-148a-3p/wnt axis have never been investigated. For instance, IncRNA NEAT1 was identified to interact with miR21/RRM2 axis in breast cancer and the axis was deemed as a novel biomarker in BC [10]. Again, the interplay between IncRNA H19/miR-675 versus IncRNA NEAT1/miR-204 axes was reported to be effective in breast cancer therapy [11]. In addition, silencing of IncRNA NEAT1 was discovered to repress colorectal cancer via regulation of microRNA miR-193a-3p/KRAS axis [12]. The migration and invasion of breast cancer cells was mediated and enhanced by miR-133b being sponged and silenced by IncRNA NEAT1 [11]. It has also been reported that NEAT1 was involved to have negatively regulated miR-218 expression [13], facilitated cellular development and invasiveness through the miR-211/HMGA2 network [14] and promoted cellular proliferation and EMT in BC progression [15]. In this context the NEAT1 has been explored to enforce oncogenic functions in breast cancer.

However, miR-148a-3p has been reported to exhibit tumor suppressive roles in breast cancer. For example, miR-148a-3p was found to interact with Melatonin as therapeutic approach to Angiogenic aspects in breast cancer [16]. Several other functions of miR-148a-3p in breast cancer have reported in Refs. [17-21]. However, the interaction between IcnRNA NEAT1 and miR$148 a-3 p$ and their mechanistic roles in breast cancer progression are yet to be known. Additionally, the $\mathrm{Wnt} / \beta$-catenin regulatory is a group of proteins involved several crucial cellular roles including stem cellular restoration and organ development. Numerous internal cell signal transduction networks are stimulated via the Wnt, particularly the Wnt/ $\beta$-catenin dependent regulatory network [22]. The dysregulation and induction of the $\mathrm{Wnt} / \beta$-catenin regulatory cascade has been characterized by a number of human cancers [23]. In this study, the miR-148a-3p was earmarked as a suppressor of the $W n t / \beta$-catenin network and breast cancer while the IncRNA NEAT1 was identified as an upstream molecule for miR-148a-3p that promoted the activation of $\mathrm{Wnt} / \beta$-catenin pathway by sponging the microRNA to advance breast cancer. However, this aforementioned was presumed to be suitable for understanding the progression of breast cancer which could eventually provide therapeutic benefits in breast cancers as a novel axis of biomarkers.

Trop J Pharm Res, May 2021; 20(5): 900 


\section{EXPERIMENTAL}

\section{Cell culture and transfection}

We utilized these human cells for Breast Cancer (MDA-MB-231, MDA-MB-436, and MDA-MB468) including the preserved normal human breast cell line (MCF-10A) that were provided by the cell bank of the Chinese Academy of Medical Science (Shanghai, China). All cell lines were multiplied in adapted RPMI-1640 substrate, with addition of 10 percent fetal bovine serum (FBS) and $200 \mu \mathrm{g} / \mathrm{L}$ of penicillin and $200 \mu \mathrm{g} / \mathrm{L}$ of streptomycin (Beyotime, Shanghai, China). The cells were nurtured at $37^{\circ} \mathrm{C}$ in a saturated environment of 5 percent CO2. The manufacturer's guideline to perform all cell culture procedures was strictly followed.

The cells were injected with Lipofectamine 2000 (Beyotime, Shanghai, China) according to the manufacturer's instructions. Transfection of MDA-MB-436, and MDA-MB-468 cells was performed utilizing Lipofectamine 3000 (Beyotime, Shanghai, China) following manufacturer's guidelines. The transfection effectiveness was measured via qRT-PCR. The $100 \mathrm{nM}$ pcDNA3.1- IncRNA-NEAT1 and $100 \mathrm{nM}$ control pcDNA3.1 vector including the $100 \mathrm{nM}$ shRNAs (sh-IncRNA-NEAT1 and sh-control) were designed and synthesized by Guangzhou Fulengen Co., Ltd, following to the manufacturer's guidelines. The miRNA mimic and respective negative controls were also provided by Guangzhou Fulengen Co., Ltd. The experiments were conducted after 48 hours following the transfection.

\section{Real Time-quantitative Polymerase Chain Reaction (RT-qPCR)}

Entire RNA was removed from human breast cancer cell lines using Beyozol mixture (\#R0011, Beyotime, Shanghai, China) then reverse transcription of $1 \mu \mathrm{g}$ RNA was done for each specimen to cDNA using BeyoRT ${ }^{\mathrm{TM}}$ cDNA First Chain Synthesis Kit (\#D7166, Beyotime, Shanghai, China) following guidelines provided by manufacturer. The qRT-PCR experiments were conducted by use of Beyofast ${ }^{T M}$ SYBR Green QPCR Mix (Beyotime, China) and associated miRNA qRT-PCR detection kit (Beyotime, China) to observe the expression of IncRNA-NEAT1 and microRNA-148a-3p expression, respectively. GAPDH was normaolized control and the $2^{-\triangle \Delta C T}$ procedure was used to estimate the comparative expression level of IncRNA-NEAt1 and microRNA-148a-3p. The experiments were conducted in triplicates Table 1 presents sequences of the primers. All primers were produced by Genelily BioTech Co., Ltd (Shanghai, China).

Table 1: Primer sequences

\begin{tabular}{ll}
\hline Name & Sequences \\
\hline $\begin{array}{l}\text { IcnRNA-NEAT1 } \\
\text { (forward) }\end{array}$ & TAATATATCCCCAGTCTAAGGCAT \\
$\begin{array}{l}\text { IncRNA-NEAT1 } \\
\text { (reverse) }\end{array}$ & AGCTTGCAGATGGAGCCC \\
$\begin{array}{l}\text { microRNA-148a-3p } \\
\text { (forward) }\end{array}$ & GAGGAAGACAGCACGTTTGGT \\
$\begin{array}{l}\text { microRNA-148a-3p } \\
\text { (reverse) }\end{array}$ & AAAGGCGCAGCGACGT \\
$\begin{array}{l}\text { GAPDH (forward) } \\
\text { GAPDH (reverse) }\end{array}$ & AGAAGGCTGGGGCTCATTTG \\
\hline
\end{tabular}

\section{CCK-8 assay}

The transfected cells at a density of $4 \times 103$ in each well were planted into 96 well multiplying dishes, and multiplied from zero to 48 hours. Cellular viability was determined by use of CCK8 kit number C0037 (Beyotime), following standard protocol specified by manufacturer. The microplate data capturing device (Molecular Devices, Sunnyvale, USA) was employed so as to quantify wave length optical density at $450 \mathrm{~nm}$.

\section{Flow Cytometry assay}

Flow cytometry assay was performed to examine the apoptosis rate. Human breast cancer cell lines MDA-MB-231 and MDA-MB-468 were transitorily transfected with pcDNA3.1-IncRNA NEAT1, control pcDNA3.1 vector, siRNAs (siIncRNA-NEAT1and si-control) including microRNA-148a-3p mimic and respective negative controls for 24 hours. Then, the preserved breast cancer cell lines MDA-MB-231 and MDA-MB-468 were cultured utilizing annexin V/PI mixture (Beyotime, Shanghai, China) for 30 minutes at room temperature, without any light.

\section{Western blotting assay}

Western blot determined the proteins concentration in autophagy related biomarkers. The obtained $B C$ cells were lysed in RIPA lysate (strong, \#P0013B, Beyotime, Shanghai, China) with protease inhibitor mixture (universal, 100X, \#P1005, Beyotime, Shanghai, China) to obtain total protein. The protein quantification was analyzed using a BeyoECL Star (Ultra-Sensitive ECL Chemical Luminescent Kit) (\#P0018AS, Beyotime, Shanghai, China), adhering to the manufacturer's protocol at $4^{\circ} \mathrm{C}$ for 10 minutes. Parts of protein were isolated by $20 \%$ SDSPAGE electrophoresis and were moved to 
polyvinylidene fluoride (PVDF) membranes (Beyotime, Shanghai, China). Then, membranes were congested in $10 \%$ scan milk/TBST (BD Biosciences, \#BYL40422, USA) and were incubated with suitable primary antibodies targeting microRNA-148a-3p (Beyotime, Shanghai, China), anti-LC3B (LC3B Rabbit Polyclonal Antibody, 1:2000, \#AF5225, Beyotime, Shanghai, China), anti- $\beta$-Catenin ( $\beta$ Catenin Rabbit Polyclonal Antibody, 1:1000, \#AF0066, Beyotime, Shanghai, China), anti-cmyc (Myc Tag Mouse Monoclonal Antibody, 1:1000, \#AF0033, Beyotime, Shanghai, China), anti-cyclin-D1(Cyclin D1 Rabbit Polyclonal Antibody, 1:2000, \#AF0126, Beyotime, Shanghai, China) and anti GAPDH (GAPDH Rabbit Monoclonal Antibody, 1:100, \#AF1186, Beyotime, Shanghai, China) at $25{ }^{\circ} \mathrm{C}$ temperature for 3 hours. The films were nurtured with horseradish peroxidase-conjugated secondary antibodies (Santa Cruz Biotechnology, Dallas, TX, USA). The protein indication was examined using the boosted chemiluminescence reagents kit (Beyotime). The image $\mathrm{J}$ detection system was employed to determine the concentration of the bands. GAPDH was normalized control. The analysis of each group was repeated in three-fold.

\section{Bioinformatics analysis}

The target connecting locations microRNA-148a$3 p$ including IncRNA-NEAT1 were analyzed Starbase.

\section{Luciferase reporter assay}

The target sequence of IcnRNA-NEAT1 and microRNA-148a-3p connecting locations were predicted and replicated into a pGL3 Dual-luciferase Target Vector (Promega, USA), to construct Wild Type and Mutant Type IncRNANEAT1 plasmids. These Wild Type or Mutant Type IncRNA-NEAT1 membranes were transfected together into MDA-MB-231 and MDA-MB-468 cell lines along with mimic-NC or microRNA-148a-3p mimic (Guangzhou Fulengen Co., Ltd, Guangzhou, China) using Lipofectamine 3000 following manufacturer's protocol. Following elapsing of $48 \mathrm{hrs}$, we conducted a luciferase assay via the dual-luciferase reporter method (Promega, USA), following protocol specified by the manufacture.

\section{Statistical analysis}

The trials were conducted in three-fold and separately. The experimental data has been exhibited as average and standard error. The GraphPad Prism 5 software and SPSS 18.0 version analyzed the statistics of the study. Student's t-test and ANOVA analysis were applied. The level of significance was P-value less than 0.05 to show a difference in statistical significance.

\section{RESULTS}

LncRNA NEAT1 was upregulated in BC cell lines and promoted cell viability, autophagy and inhibited Apoptosis in BC cells

The RT-qPCR measured the expression of NEAT1 which was analyzed in three separate human breast cancer cell lines (MDA-MB-231, MDAMB-436, and MDA-MB-468) and a single normal breast epithelial cell line (MCF-10A). The outcomes indicated elevated expressions of NEAT1 in the human BC cell lines (MDA-MB231, MDAMB-436, and MDA-MB-468) in contrast to the epithelial normal breast cell line which indicated very low expression (Figure 1A, $p<0.5$ ). The increments in expression by NEAT1 in human $B C$ cell lines were notably higher in the MDAMB-436, and MDA-MB-468 cell lines that were adopted for further experiments. Then, the IncRNA-NEAT1 was overexpressed by pcDNA3.1 and RT-qPCR measured the efficacy of the expression in both adopted cell lines (Figure 1B, p<0.5). The results if Fig.1B showed that IncRNA-NEAT1 was upregulated in the pcDNA3.1 IcnRNA-NEAT1 in contrast to the negative control. Then, cellular viability, apoptosis and autophagy were detected via CCK-8, FCM and western blot assays respectively in both MDAMB-436, and MDA-MB468 cell lines. Thereafter, the outcomes indicated increased cellular viability in the NEAT1 overexpressed group in contrast to the control group (Figure 1C, $\mathrm{p}<0.5$ ). However, the apoptosis rate was decreased after NEAT1 was upregulated in comparison to the negative control (Fig.1D, p<0.5). For autophagy, the western blot results indicated increased level of expression in related biomarkers (LC3B-I and LC3B-II) in both adopted cell lines for upregulated NEAT1 compared to negative control group (Figure 1E, $\mathrm{p}<0.5$ ). In general, these outcomes signify the influence of IncRNANEAT1 on cellular viability, apoptosis and autophagy when upregulated.

LncRNA NEAT1 knockdown inhibited cell viability, autophagy and enhanced apoptosis

In order to understand further the effect of low levels of NEAT1 on cell viability, autophagy and apoptosis, NEAT1 was silenced and the knockdown efficacy was determined by RTqPCR (Figure 2A, $p<0.5$ ). As shown in Figure 2A, 


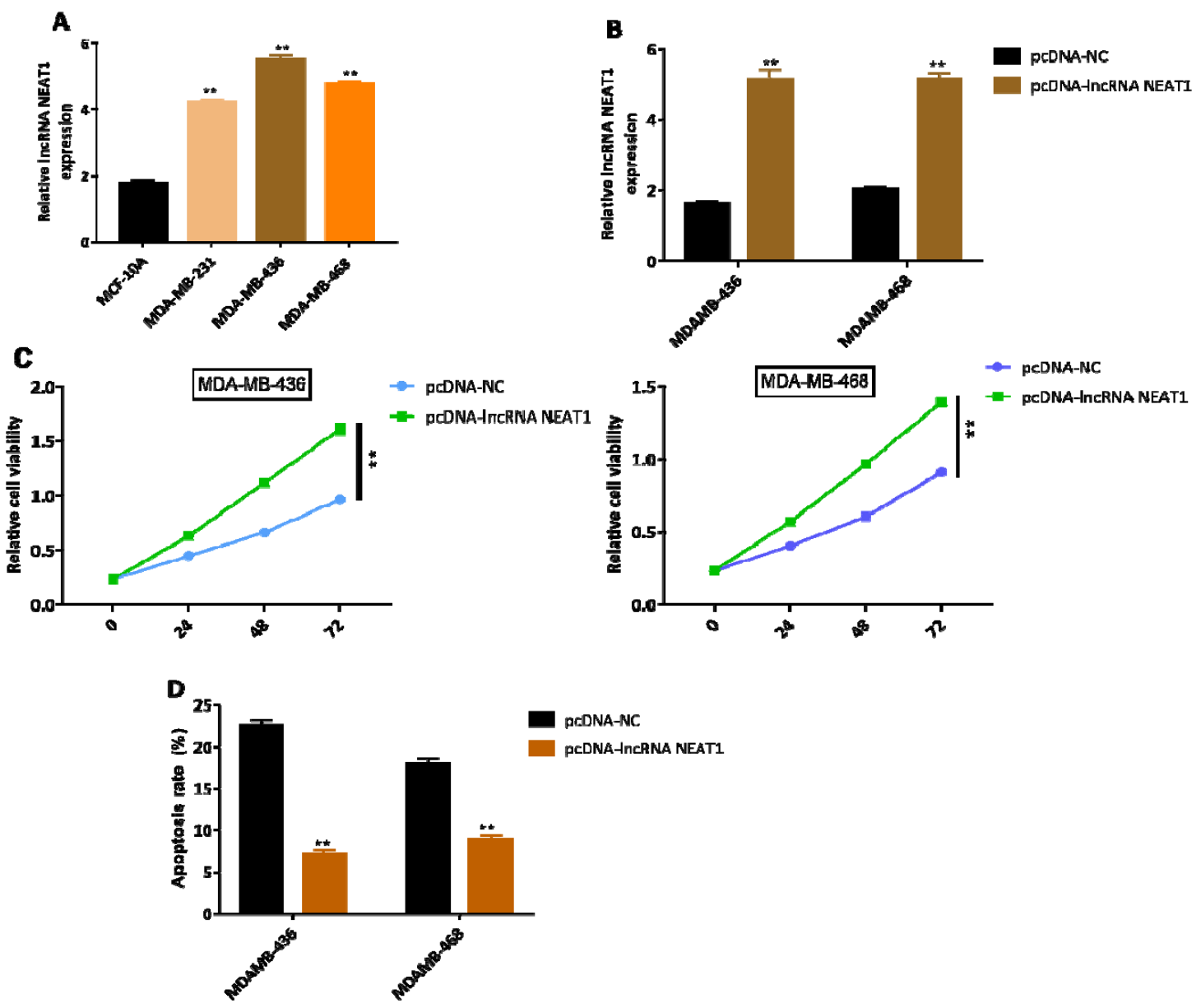

Figure 1: LncRNA NEAT1 was upregulated in BC cell lines and promoted cell viability, autophagy and inhibited Apoptosis in BC cells.

A) RT-qPCR assay measured IncRNA NEAT1 expression in three separate human Breast Cancer cell lines (MDA-MB-231, MDAMB-436, and MDA-MB-468) and a single normal breast epithelial cell line (MCF-10A) $(p<0.05)$. B) RT-qPCR detected overexpression efficacy of IncRNA NEAT1 in MDAMB-436 and MDA-MB-468 cell lines (p<0.05). C) CCK-8 assay measure cellular viability of MDAMB-436 and MDA-MB-468 cells after IncRNA NEAT1-overexpression ( $p<0.05)$. D) FCM assay measured apoptosis rate in MDAMB-436 and MDA-MB-468 cells after IncRNA NEAT1-overexpression ( $p<0.05)$. All data were Mean and $S D$

the NEAT1 expression was remarkably reduced in the silenced group in comparison to the control group. Next, cellular viability, apoptosis and autophagy were detected via CCK-8, FCM and western blot assays respectively in both MDAMB-436, and MDA-MB-468 cell lines. The CCK-8 outcomes indicated remarkable decrease in cellular viability in the NEAT1 silenced group in contrast to the control group in both cell lines (Figure 2B, $\mathrm{p}<0.5$ ). Further, the apoptosis rate dramatically increased following NEAT1 knockdown compared with negative control in both cell lines (Figure 2C, $\mathrm{p}<0.5$ ). For autophagy, western blot outcomes indicated dramatic decline in the autophagy related biomarkers (LC3B-I and LC3B-II) for the silenced NEAT1 group contrasting to the negative control in both adopted cell lines (Figure 2D, $p<0.5$ ). In general, these outcomes imply that knocking down NEAT1 exhibits inhibitory effects on cellular viability and autophagy but enhances apoptosis.

\section{LncRNA NEAT1 directly targets microRNA- 148a-3p}

To comprehend the relationship among IncRNANEAT1 and microRNA-148a-3p, we performed a prediction analysis via bioinformatics tool Starbase. It was predicted that these two genes have some binding locations in which microRNA148a-3p was connecting with IncRNA-NEAT1 (Figure $3 A$ ). To further validate this interaction, the luciferase reporter assay was performed. First, we created and generated luciferase reporter for wild type IncRNA-NEAT1 (NEAT1WT) or mutant type IncRNA-NEAT1 (NEAT1- 


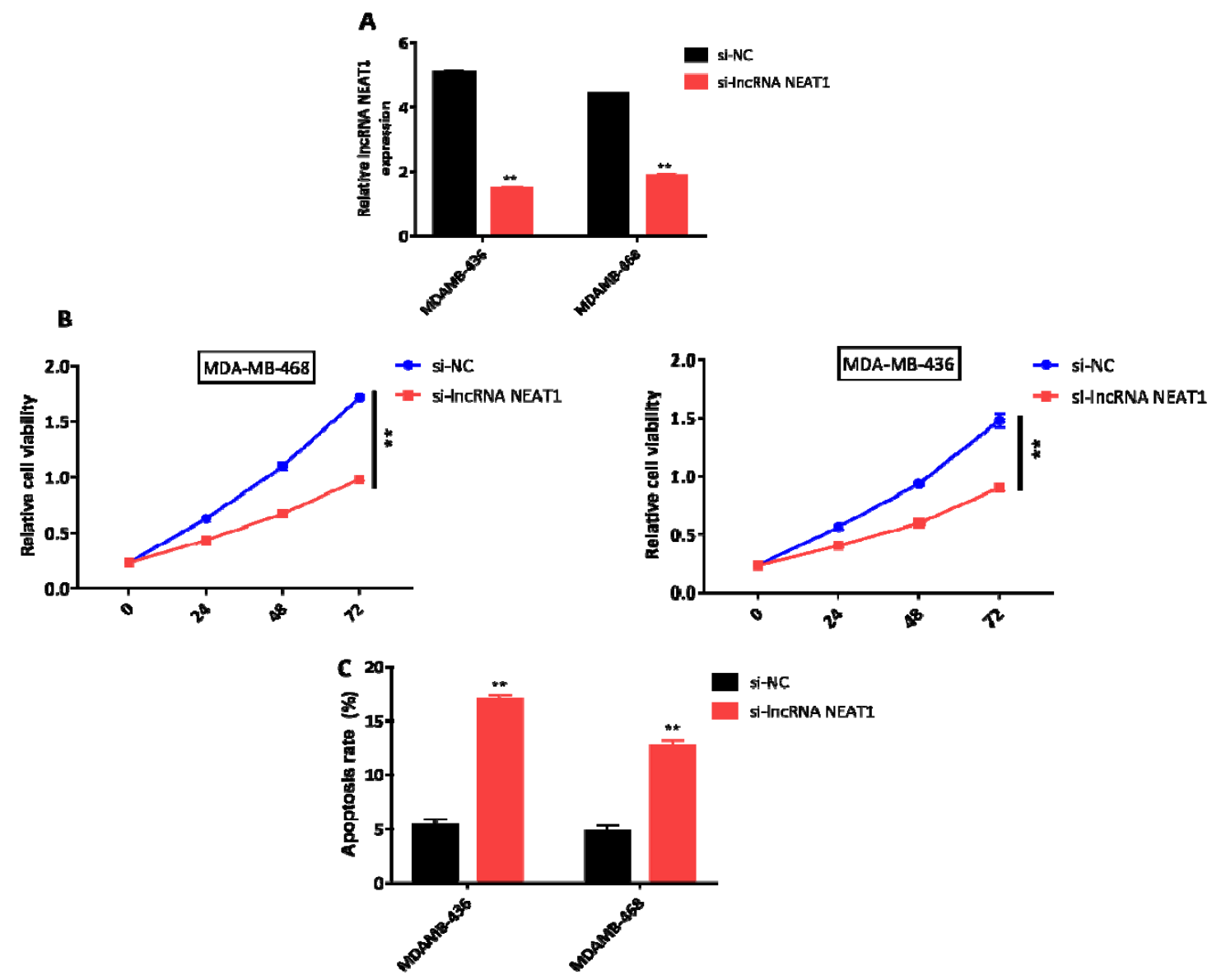

Figure 2: LncRNA NEAT1 knockdown inhibited cell viability, autophagy and enhanced apoptosis.

A) RT-qPCR assay measured IncRNA NEAT1 knock down expression efficacy in human Breast Cancer cell lines (MDAMB-436 and MDA-MB-468) (p<0.05). B) CCK-8 assay measure cellular viability of MDAMB-436 and MDAMB-468 cells after IncRNA NEAT1-knock down $(p<0.05)$. C) FCM assay measured apoptosis rate in MDAMB436 and MDA-MB-468 cells after IncRNA NEAT1-knock down $(p<0.05)$. All data were Mean and SD

MUT) followed by transfection with either negative controls or microRNA-148a-3p mimic in $\mathrm{BC}$ cell lines. The results revealed that silenced IncRNA-NEAT1 led to decreased luciferase activity in NEAT1-WT transfected with microRNA-148a-3p mimic compared with negative control in both $\mathrm{BC}$ cells (Figure $3 \mathrm{~B}$, p<0.5).. However, with no activity observed in NEAT1-MUT transfected with microRNA-148a-3p mimic compared with negative control in both $B C$ cells (Figure $3 \mathrm{C}, \mathrm{p}<0.05$ ). These data implied that IncRNA-NEAT1 might directly sponge microRNA-148a-3p in BC. Lastly, the expression of microRNA-148a-3p was detected via RTqPCR in in three separate human Breast Cancer cell lines (MDA-MB-231, MDAMB-436, and MDAMB-468) and a single normal breast epithelial cell line (MCF-10A). The outcomes indicated significantly lowered expressions of microRNA$148 a-3 p$ in the human BC cell lines (MDA-MB231, MDAMB-436, and MDA-MB-468) in contrast to the epithelial normal breast cell line which indicated very high expression (Figure 3D, $p<0.5)$. The decrease in expression by microRNA-148a-3p in human $B C$ cell lines were notably lower in the MDAMB-436 and MDA-MB468 cell lines that were adopted for further experiments.

MicroRNA-148a-3p overturns the cellular viability, autophagy and inhibitory effects on Apoptosis by IncRNA-NEAT1

To further understand the interplay between IncRNA-NEAT and microRNA-148a-3p on how they influence cell viability, autophagy and apoptosis if they are interacting, we performed CCK-8, western blot and FCM assays respectively. Firstly, the $\mathrm{BC}$ cell lines were transfected with either pcDNA-NC, pcDNAIncRNA-NEAT1+microRNA-148a-3p mimic or pcDNA-IncRNA-NEAT1 followed by RT-qPCR to 
A

Target: $5^{\prime}$ ggacuGUUCUGUCCUUGCACUGc $3^{\prime}$

| ||||| |||||||

miRNA : 3' UguUuCAAGACA-UCACGUGACu $5^{\prime}$
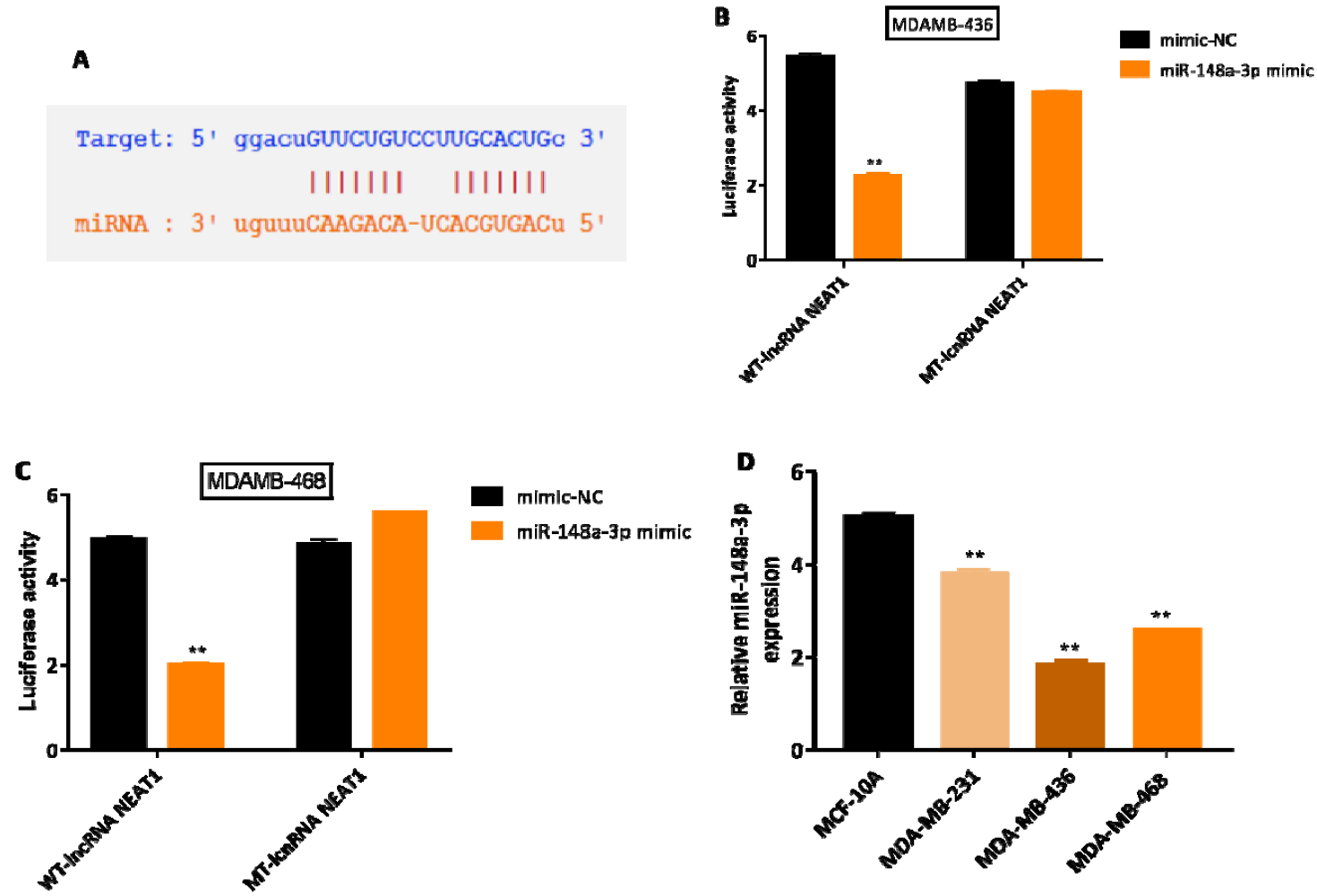

Figure 3: LncRNA NEAT1 directly targets microRNA-148a-3p

A) Bioinformatics tool Starbase predicted the connecting location of IncRNA-NEAT1 and microRNA-148a-3p. B) $B$.Luciferase reporter assay to measure luciferase activity in MDAMB-436 cell line $(p<0.05)$. C) Luciferase reporter assay to measure luciferase activity in MDAMB-468 cell line $(p<0.05)$. D) $R T-q P C R$ assay measured microRNA-148a-3p expression in three separate human Breast Cancer cell lines (MDA-MB-231, MDAMB-436, and MDA-MB-468) and a single normal breast epithelial cell line $(M C F-10 A)(p<0.05)$. All data were Mean and $S D$

evaluate expression efficiency of microRNA148a-3p (Figure 4A, $p<0.5$ ). As shown in the Figure, the results showed highest expression in the overexpressed group (pcDNA-IncRNANEAT1) compared to the lower expression in the control group (pcDNA-NC) in both cell lines. However, Figure 4A also shows that combined interplay between co-transfected overexpressedIncRNA-NEAT1 with microRNA-148a-3p mimic led to a remarkable decline in expression contrasting to the overexpressed group (pcDNAIncRNA-NEAT1) in both cell lines. Furthermore, the CCK-8 assay outcomes demonstrated dramatic decreased cell viability in the cotransfected overexpressed-IncRNA-NEAT1 with microRNA-148a-3p mimic compared to the IncRNA-NEAT1 overexpressed group and negative control group in both cell lines (Figure $4 \mathrm{~B}, \mathrm{p}<0.5)$. In addition, the apoptosis rate was remarkably higher in the co-transfected overexpressed-IncRNA-NEAT1 with microRNA148a-3p mimic compared to the IncRNA-NEAT1 overexpressed group and negative control group in both cell lines (Figure 4C, $p<0.5$ ). For autophagy, the western blot results indicated lower levels of LC3B-I and LC3B-II in the cotransfected overexpressed-IncRNA-NEAT1 with microRNA-148a-3p mimic compared to the IncRNA-NEAT1 overexpressed group and negative control group in both cell lines (Figure $4 D, p<0.5)$. Generally, these results suggest that microRNA-148a-3p reversed the cellular viability, autophagy and inhibitory effects on Apoptosis by IncRNA-NEAT1.

\section{LncRNA NEAT1 activated the Wnt/ $\beta$-catenin regulatory network through sponging microRNA-148a-3p in BC cell lines}

The Wnt/ $\beta$-catenin regulatory pathway is an essential moderator of tumor development and advancement. Unsurprisingly, the microRNA and protein quantities in various lower-level targets of the $W n t / \beta$-catenin regulatory network, as well as $\beta$-catenin, c-myc plus cyclinD1, were dramatically increased in the IncRNA NEAT1overexpressed in both MDAMB-436 and MDA- 

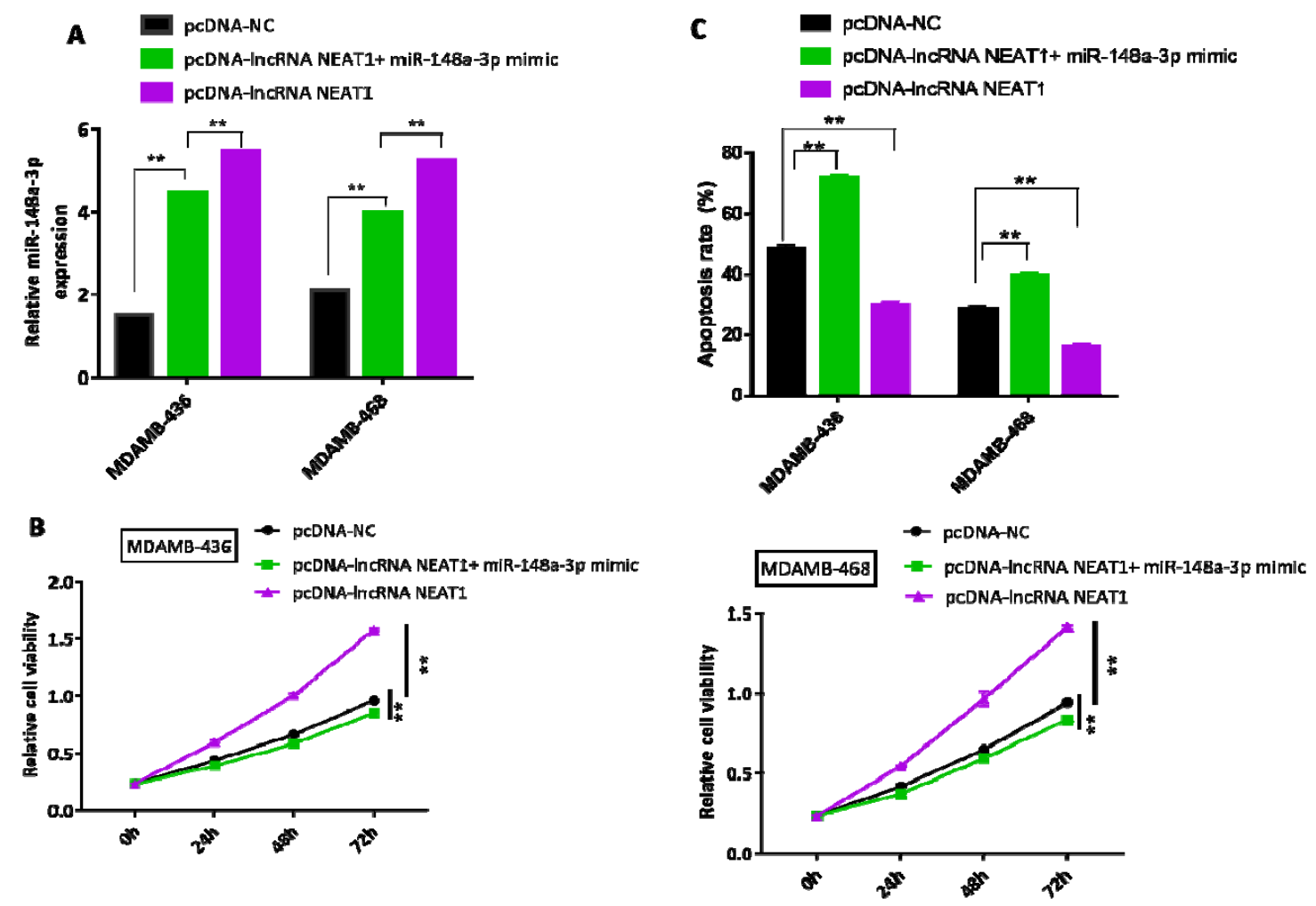

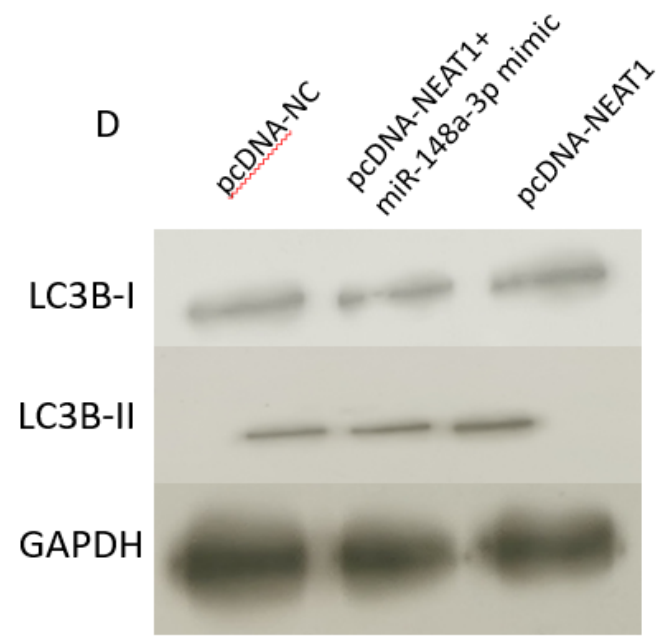

MDAMB-436

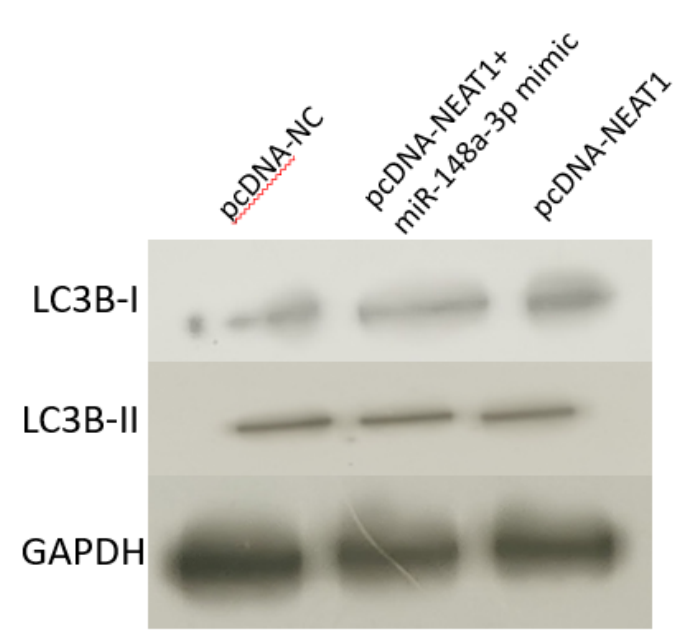

MDA-MB-468

Figure 4: MicroRNA-148a-3p overturns the cellular viability, autophagy and inhibitory effects on Apoptosis by InCRNA-NEAT1.

A) RT-qPCR detected expression efficacy of microRNA-148a-3p in MDAMB-436 and MDA-MB-468 cell lines for either of these groups ( $p c D N A-N C$, pcDNA-IncRNA NEAT1+ miR-148a-3p mimic or pcDNA-IncRNA NEAT1) $(p<0.05)$. B) CCK-8 assay measure cellular viability of microRNA-148a-3p expression in MDAMB-436 and MDA$M B-468$ cell lines for either of these groups ( $p c D N A-N C$, $p c D N A-I n c R N A N E A T 1+$ miR-148a-3p mimic or pcDNAIncRNA NEAT1) ( $p<0.05)$. C) FCM assay measured apoptosis rate of microRNA-148a-3p expression in MDAMB436 and MDA-MB-468 cell lines for either of these groups ( $p c D N A-N C$, pcDNA-IncRNA NEAT1+ miR-148a-3p mimic or pcDNA-IncRNA NEAT1) ( $p<0.05)$. D) Western blot examined autophagy based on microRNA-148a-3p expression in MDAMB-436 and MDA-MB-468 cell lines for either of these groups ( $p c D N A-N C$, pcDNA-IncRNA NEAT1+ miR-148a-3p mimic or pcDNA-IncRNA NEAT1) ( $p<0.05)$. All data were Mean and $S D$ 
MB-468 BC cell lines and reduced in the negative control group in both MDAMB-436 and MDA-MB-468 BC cell lines. Nonetheless, cotransfecting overexpressed-IncRNA-NEAT1 with microRNA-148a-3p mimic group overturned the hyper invigorating of the Wnt/ $\beta$-catenin regulatory network in the overexpressed-IncRNANEAT1 transfected cells (Figure $5, p<0.05$ ). To sum up, these outcomes evidently proved that overexpressing IncRNA-NEAT1 hyper stimulated the $W n t / \beta$-catenin regulatory network via sponging the microRNA-148a-3p in breast cancer cells.

\section{DISCUSSION}

The breast cancer has been reported to be among the extremely belligerent melanomas resulting from numerous factors [24]. By means of speedy advances in molecular biotechnology, biochemical motivated treatment has been increasingly employed to aid in crucial therapy for breast cancer [25]. A large body of evidence suggests that dysregulated expression of IncRNAs is involved in several malignancies, and the IncRNA motivated target therapies have been applied as new detection, prediction and treatment tools in BC. For example other researchers have investigated the mechanistic roles of IncRNAs such as NKILA [26], DANCR [27], GIHCG [28], TTN-AS1 [29], LINC02273 [30] and DLEU1 [31] in breast cancer among others. To the best of the authors' knowledge, this study offers firsthand knowledge and information for the first time in relation to the novel mechanistic link among NEAT1, microRNA-148a-3p and the $W n t / \beta$-catenin regulatory network in human Breast Cancer. We discovered that IncRNANEAT1 expression was dramatically elevated in $B C$ cell lines. In addition, upregulation of IncRNANEAT1 expression accelerated cellular viability, autophagy and prevented apoptosis while downregulation of IncRNA-NEAT1 expression repressed, Breast Cancer cellular viability, autophagy and promoted apoptosis in vitro. Furthermore, it has been shown in this study that IncRNA-NEAT1 targeted microRNA-148a-3p which was lowly expressed in BC. And, the interaction among IncRNA NEAT1 and microRNA-148a-3p elucidated that this interplay results in dramatic reduces expression levels in IncRNA which in turn reverses the effects of cellular proliferation, autophagy enhancement and apoptosis inhibition as influenced by IncRNA NEAT1 overexpression. Moreover, the authors have demonstrated that overexpressed IncRNANEAT1 hyper invigorated the Wnt/ß-catenin regulatory network through acting as a direct sponge to microRNA-148a-3p. Jointly, these outcomes have showcased that IncRNA NEAT1 acts as malignant promoter or tumor oncogene in breast cancer and may perhaps be considered as an essential therapeutic target for $\mathrm{BC}$ in medical treatment.

The recognized $W n t / \beta$-catenin network is activated through the released Wnt ligands that connect with Frizzled (FZD) group conduit including LRP5/LRP6 co-receptor to incite the $\beta$ catenin network structure, eventually expedites the expression of c-myc and cyclin-D1 [32]. Deregulated invigoration of the $\mathrm{Wnt} / \beta$-catenin regulatory network is frequently discovered in several human melanomas and may possibly advance tumor growth with BC inclusive [33]. Thus, comprehending the fundamental mechanisms is essential for the advancement and perfection in cancer treatment. Cui et al. discovered that LncRNA Neat1 mediated microRNA-124-stimulated activation of $\mathrm{Wnt} / \beta$ catenin network to regulate neuronal differentiation, apoptosis and migration in spinal cord neural progenitor [34]. Thus, their study gave precedential illustrative fundamental molecular mechanistic functions of IncRNA and Wnt/ $\beta$-catenin network that could be regarded a potential treatment approach even for cancer. Nonetheless, so far, the upstream features of the Wnt/ $\beta$-catenin regulatory network together with the fundamental roles in the regulation of carcinogenesis and metastasis in breast cancer is yet to be clarified extensively. De-regulated IncRNAs' expressions play essential functions in initiation of cancer and subsequent advancement, and the controlling mechanisms of IncRNAs in carcinomas are far much appealing to the scientific community worldwide. Preceding papers have recognized the oncogenic role of IncRNA NEAT1 when it is overexpressed in several human cancers including Hepatocellular Carcinoma [35, 36]. Intriguingly, silenced LncRNA NEAT1 moderates autophagy to increase 5-FU reaction in colorectal cancer through targeting microRNA-34a [37]. In this investigation, it has been demonstrated through the outcomes that overexpressed IncRNANEAT1 might enhance cellular viability, autophagy and prevent apoptosis in breast cancer cells via invigorating the Wnt/ $\beta$-catenin regulatory network by sponging microRNA-148a$3 p$. Other studies have showcased that IncRNANEAT1 played the role of a competitive endogenous RNA to; microRNA-410 in bladder cancer [38], microRNA-214 in multiple myeloma [39] including microRNA-124 and microRNA$125 \mathrm{a}$ in acute ischemic stroke [40]. Some of the functional molecular roles in these studies indicated regulation of M2 macrophage polarization, enhancement of disease risk, poor recurrence-free survival of patients and 
A
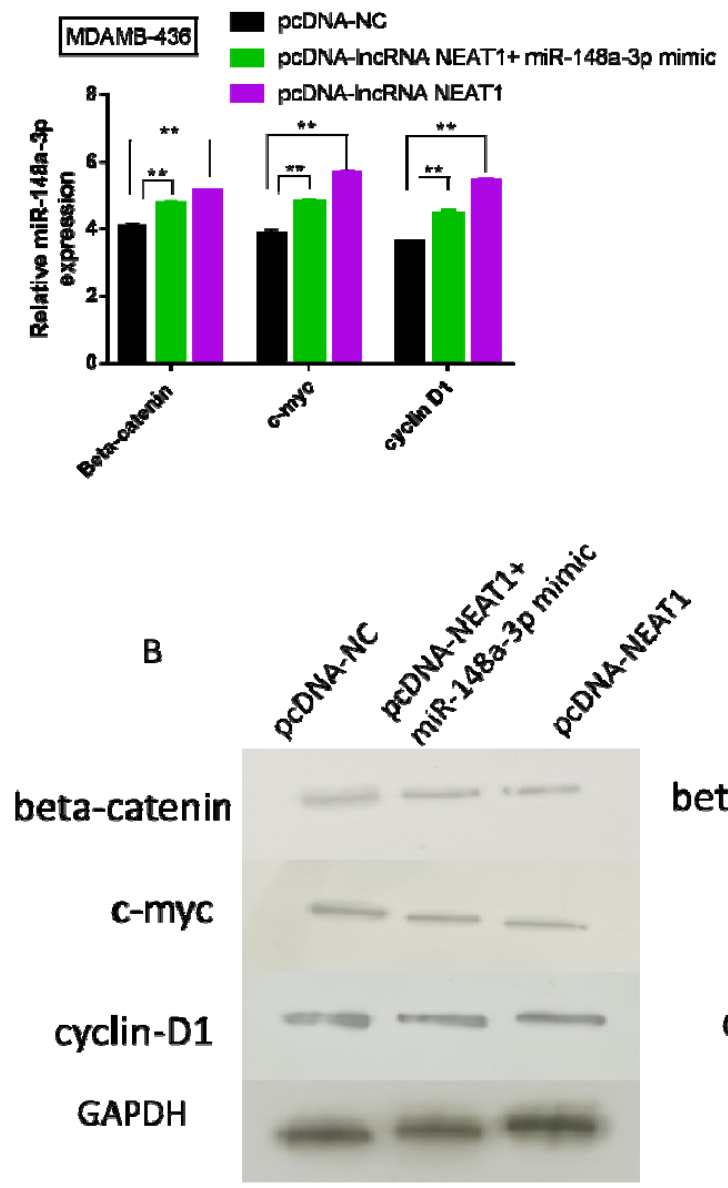

MDAMB-436

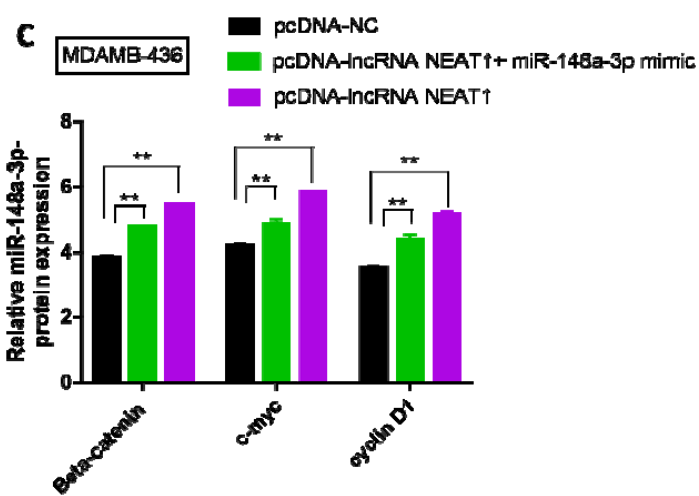

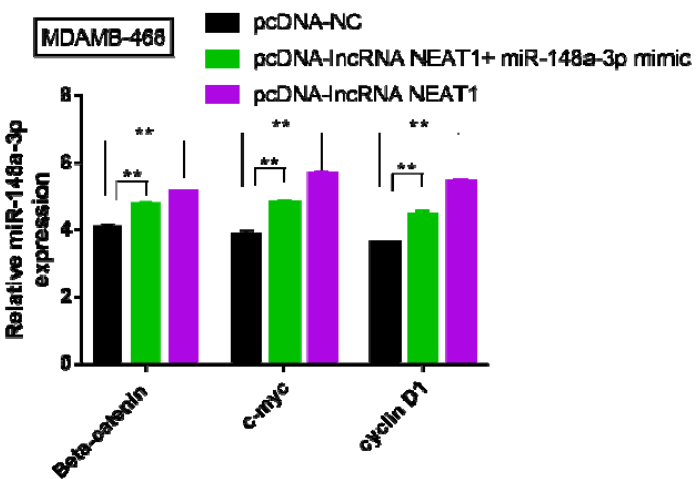

beta-catenin

c-myc

cyclin-D1

GAPDH

MDA-MB-468

Figure 5: LncRNA NEAT1 activated the Wnt/ $\beta$-catenin regulatory network through sponging microRNA-148a-3p in $\mathrm{BC}$ cell lines

A) RT-qPCR detected relative expression of miRNA in beta-catenin, c-myc and cyclin-D1 in MDAMB-436 and MDA-MB-468 cell lines for either of these groups (pcDNA-NC, pcDNA-IncRNA NEAT1+ miR-148a-3p mimic or pcDNA-IncRNA NEAT1) $(p<0.05)$. B) Western blot assay measured relative miRNA protein expression in betacatenin, c-myc and cyclin-D1 in MDAMB-436 and MDA-MB-468 cell lines for either of these groups (pcDNA-NC, pcDNA-IncRNA NEAT1+ miR-148a-3p mimic or pcDNA-IncRNA NEAT1) $(p<0.05)$. C) RT-qPCR detected protein expression of miRNA in beta-catenin, c-myc and cyclin-D1 in MDAMB-436 and MDA-MB-468 cell lines for either of these groups (pcDNA-NC, pcDNA-IncRNA NEAT1+ miR-148a-3p mimic or pcDNA-IncRNA NEAT1) $(p<0.05)$. All data were Mean and SD 
development and progression of these diseases. Thus, direct target of IncRNA-NEAT1 was further explored in $\mathrm{BC}$.

In this study the binding location of IncRNA NEAT1 and microRNA-148a-3p were predicted by application of bioinformatics analytics using Starbase second version (2.0). Meanwhile, the outcomes from the experiments showed that overexpression of IncRNA-NEAT1 prevent the expression of microRNA-148a-3p while it's silencing led it increased expression of microRNA-148a-p. In addition, microRNA-148a$3 p$ expression co-transfected with overexpressed IncRNA-NEAT1 reversed proliferation effects and overturned enhanced autophagy coupled with improved apoptosis. Thus, we suggest that IncRNA-NEAT1 might directly sponge the microRNA-148a-3p and eventually activates the Wnt network in breast cancer. To this end, this paper has preliminarily elucidated the fundamental correlation among InchRNA-NEAT1 and microRNA-148a-3p. So far, the molecular mechanistic roles fundamental to the pathogenesis of breast cancer still remain vague.

\section{CONCLUSION}

The present study showcased that IncRNANEAT1 could enhance tumor development in breast cancer via playing the role of molecular sponge to microRNA-148a-3p, and eventually hyper invigorating the $\mathrm{Wnt} / \beta$-catenin regulatory network. These outcomes unearth a novel biomarker of the Wnt network hyper stimulation in Breast Cancer, and IncRNA NEAT1 could be a prospective treatment tool for BC therapy in the immediate time ahead.

\section{DECLARATIONS}

\section{Conflict of interest}

No conflict of interest is associated with this work.

\section{Contribution of authors}

We declare that this work was done by the authors named in this article and all liabilities pertaining to claims relating to the content of this article will be borne by the authors.

\section{Open Access}

This is an Open Access article that uses a funding model which does not charge readers or their institutions for access and distributed under the terms of the Creative Commons Attribution
License (http://creativecommons.org/licenses/by/ 4.0) and the Budapest Open Access Initiative (http://www.budapestopenaccessinitiative.org/rea d), which permit unrestricted use, distribution, and reproduction in any medium, provided the original work is properly credited.

\section{REFERENCES}

1. Hu K, Ding P, Wu Y, Tian W, Pan T, Zhang S: Global patterns and trends in the breast cancer incidence and mortality according to sociodemographic indices: an observational study based on the global burden of diseases. BMJ open 2019, 9:e028461.

2. DeSantis C, Ma J, Bryan L, Jemal A: Breast cancer statistics, 2013. CA: a cancer journal for clinicians 2014, 64:52-62.

3. $\mathrm{Li} \mathrm{T}$, Mello-Thoms C, Brennan PC: Descriptive epidemiology of breast cancer in China: incidence, mortality, survival and prevalence. Breast cancer research and treatment 2016, 159:395-406.

4. Ye DM, Li Q, Yu T, Wang HT, Luo YH, Li WQ: Clinical and epidemiologic factors associated with breast cancer and its subtypes among Northeast Chinese women. Cancer medicine 2019, 8:7431-7445.

5. $A M B, R V S, J M E, A$ AO: Breast cancer biomarkers: risk assessment, diagnosis, prognosis, prediction of treatment efficacy and toxicity, and recurrence. Current pharmaceutical design 2014, 20:4879-4898.

6. Jafari SH, Saadatpour Z, Salmaninejad A, Momeni F, Mokhtari M, Nahand JS, Rahmati M, Mirzaei $H$, Kianmehr M: Breast cancer diagnosis: Imaging techniques and biochemical markers. Journal of cellular physiology 2018, 233:5200-5213.

7. Zhou W, Gong J, Chen Y, Chen J, Zhuang Q, Cao J, Mei Z, Hu B: Long noncoding RNA LINC00899 suppresses breast cancer progression by inhibiting miR-425. Aging 2019, 11:10144-10153.

8. Li J, Guo W, Xue W, Xu P, Deng Z, Zhang $D$, Zheng $S$, Qiu X: Long noncoding RNA AURKAPS1 potentiates malignant hepatocellular carcinoma progression by regulating miR-142, miR-155 and miR-182. Scientific reports 2019, 9:19645.

9. Sun C, Zhou Z, Shi H, Li F, Zhang G: Identification of Long Noncoding RNA APOC1P1 as an Oncogene in Clear Cell Renal Cell Carcinoma. Disease markers 2019, 2019:2814058.

10. Quan D, Chen K, Zhang J, Guan Y, Yang D, Wu H, Wu S, LV L: Identification of IncRNA NEAT1/miR-21/RRM2 axis as a novel biomarker in breast cancer. Journal of cellular physiology 2019.

11. Muller V, Oliveira-Ferrer L, Steinbach B, Pantel K, Schwarzenbach H: Interplay of IncRNA H19/miR-675 and IncRNA NEAT1/miR-204 in breast cancer. Molecular oncology 2019, 13:1137-1149.

12. Zhu Z, Du S, Yin K, Ai S, Yu M, Liu Y, Shen Y, Liu M, Jiao $R$, Chen X, Guan W: Knockdown long noncoding RNA nuclear paraspeckle assembly transcript 1 suppresses colorectal cancer through modulating miR-193a3p/KRAS. Cancer medicine 2019, 8:261-275.

13. Zhao $D$, Zhang $Y$, Wang $N$, Yu N: NEAT1 negatively regulates miR-218 expression and promotes breast cancer progression. Cancer biomarkers : section $A$ of Disease markers 2017, 20:247-254.

14. Li X, Wang S, Li Z, Long X, Guo Z, Zhang G, Zu J, Chen $Y$, Wen $L$ : The IncRNA NEAT1 facilitates cell growth and invasion via the miR-211/HMGA2 axis in breast cancer. International journal of biological macromolecules 2017, 105:346-353.

15. Zhang $M$, Wu WB, Wang ZW, Wang XH: IncRNA NEAT1 is closely related with progression of breast cancer via promoting proliferation and EMT. European review for 
medical and pharmacological sciences 2017, 21:10201026.

16. Lacerda JZ, Ferreira LC, Lopes BC, Aristizabal-Pachon AF, Bajgelman MC, Borin TF, Zuccari D: Therapeutic Potential of Melatonin in the Regulation of MiR-148a$3 p$ and Angiogenic Factors in Breast Cancer. MicroRNA (Shariqah, United Arab Emirates) 2019, 8:237-247.

17. Chen $X$, Wang YW, Gao P: SPIN1, negatively regulated by miR-148/152, enhances Adriamycin resistance via upregulating drug metabolizing enzymes and transporter in breast cancer. Journal of experimental \& clinical cancer research : CR 2018, 37:100.

18. Idichi T, Seki $N$, Kurahara $H$, Fukuhisa $H$, Toda $H$ Shimonosono M, Okato A, Arai T, Kita Y, Mataki Y, et al: Molecular pathogenesis of pancreatic ductal adenocarcinoma: Impact of passenger strand of premiR-148a on gene regulation. Cancer science 2018, 109:2013-2026.

19. Simpson MR, Brede G, Johansen J, Johnsen R, Storro O, Saetrom $P$, Oien T: Human Breast Milk miRNA Maternal Probiotic Supplementation and Atopic Dermatitis in Offspring. PloS one 2015, 10:e0143496.

20. Zeng K, He B, Yang BB, Xu T, Chen X, Xu M, Liu X, Sun $H$, Pan $Y$, Wang S: The pro-metastasis effect of circANKS1B in breast cancer. Molecular cancer 2018, 17:160.

21. Ashizawa M, Okayama H, Ishigame T, Thar Min AK, Saito K, Ujiie D, Murakami Y, Kikuchi T, Nakayama Y, Noda $M$, et al: miRNA-148a-3p Regulates Immunosuppression in DNA Mismatch Repair-Deficient Colorectal Cancer by Targeting PD-L1. Molecular cancer research : MCR 2019, 17:1403-1413.

22. Krishnamurthy N, Kurzrock R: Targeting the Wnt/betacatenin pathway in cancer: Update on effectors and inhibitors. Cancer treatment reviews 2018, 62:50-60.

23. Wang Z, Li B, Zhou L, Yu S, Su Z, Song J, Sun Q, Sha O, Wang $X$, Jiang $W$, et al: Prodigiosin inhibits Wnt/betacatenin signaling and exerts anticancer activity in breast cancer cells. Proceedings of the National Academy of Sciences of the United States of America 2016, 113:13150-13155.

24. Wheler JJ, Atkins JT, Janku F, Moulder SL, Yelensky R, Stephens PJ, Kurzrock R: Multiple gene aberrations and breast cancer: lessons from super-responders. BMC cancer 2015, 15:442.

25. Zaidat OO, Fitzsimmons BF, Woodward BK, Wang Z Killer-Oberpfalzer M, Wakhloo A, Gupta R, Kirshner H, Megerian JT, Lesko J, et al: Effect of a balloonexpandable intracranial stent vs medical therapy on risk of stroke in patients with symptomatic intracranial stenosis: the VISSIT randomized clinical trial. Jama 2015, 313:1240-1248.

26. Luo LH, Rao L, Luo LF, Chen K, Ran RZ, Liu XL: Long non-coding RNA NKILA inhibited angiogenesis of breast cancer through NF-kappaB/IL-6 signaling pathway. Microvascular research 2019:103968.

27. Zhang KJ, Tan XL, Guo L: The long non-coding RNA $D A N C R$ regulates the inflammatory phenotype of breast cancer cells and promotes breast cancer progression via EZH2-dependent suppression of SOCS3 transcription. Molecular oncology 2019.
28. Fan $L Y$, Shi KY, Xu D, Ren LP, Yang P, Zhang L, Wang $F$ Shao GL: LncRNA GIHCG regulates microRNA-1281 and promotes malignant progression of breast cancer. European review for medical and pharmacological sciences 2019, 23:10842-10850.

29. Qiu P, Dou Y, Ma LZ, Tang XX, Liu XL, Chen JW: Long non-coding RNA TTN-AS1 promotes the metastasis in breast cancer by epigenetically activating DGCR8. European review for medical and pharmacological sciences 2019, 23:10835-10841.

30. Xiu B, Chi Y, Liu L, Chi W, Zhang Q, Chen J, Guo R, Si J, Li L, Xue J, et al: LINC02273 drives breast cancer metastasis by epigenetically increasing AGR2 transcription. Molecular cancer 2019, 18:187.

31. Wang C, Xie $X X, L i \quad W J$, Jiang $D Q:$ LncRNA DLEU1/microRNA-300/RAB22A axis regulates migration and invasion of breast cancer cells. European review for medical and pharmacological sciences 2019, 23:10410-10421.

32. Nusse $R$, Clevers $H$ : Wnt/beta-Catenin Signaling, Disease, and Emerging Therapeutic Modalities. Cell 2017, 169:985-999.

33. Hwang MS, Yu N, Stinson SY, Yue P, Newman RJ, Allan $B B$, Dornan D: miR-221/222 targets adiponectin receptor 1 to promote the epithelial-to-mesenchymal transition in breast cancer. PloS one 2013, 8:e66502.

34. Cui Y, Yin Y, Xiao Z, Zhao Y, Chen B, Yang B, Xu B, Song $H$, Zou Y, Ma X, Dai J: LncRNA Neat1 mediates miR124-induced activation of Wnt/beta-catenin signaling in spinal cord neural progenitor cells. Stem cell research \& therapy 2019, 10:400.

35. Li Y, Ding X, Xiu S, Du G, Liu Y. LncRNA NEAT1 Promotes Proliferation, Migration And Invasion Via Regulating miR-296-5p/CNN2 Axis In Hepatocellular Carcinoma Cells. OncoTargets and therapy 2019, 12:9887-9897.

36. Zhou $X$, Wang $X$, Zhou $Y$, Cheng L, Zhang $Y$, Zhang $Y$ : Long Noncoding RNA NEAT1 Promotes Cell Proliferation And Invasion And Suppresses Apoptosis In Hepatocellular Carcinoma By Regulating miRNA-22$3 p / a k t 2$ In Vitro And In Vivo. OncoTargets and therapy 2019, 12:8991-9004.

37. Liu F, Ai FY, Zhang DC, Tian L, Yang ZY, Liu SJ: LncRNA NEAT1 knockdown attenuates autophagy to elevate 5FU sensitivity in colorectal cancer via targeting miR34a. Cancer medicine 2019.

38. Shan G, Tang T, Xia Y, Qian HJ: Long non-coding RNA NEAT1 promotes bladder progression through regulating miR-410 mediated HMGB1. Biomedicine \& pharmacotherapy = Biomedecine \& pharmacotherapie 2020, 121:109248.

39. Gao Y, Fang $P$, Li WJ, Zhang J, Wang GP, Jiang DF Chen FP: LncRNA NEAT1 sponges miR-214 to regulate $M 2$ macrophage polarization by regulation of B7-H3 in multiple myeloma. Molecular immunology 2020, 117:20-28.

40. Li P, Duan S, FU A: Long noncoding RNA NEAT1 correlates with higher disease risk, worse disease condition, decreased miR-124 and miR-125a and predicts poor recurrence-free survival of acute ischemic stroke. Journal of clinical laboratory analysis 2019:e23056. 